\title{
Influence of Hypothermia on the Clinical and Molecular Status of a Freshwater Drowning Victim with Severe Trauma. A Case Report
}

\author{
Ovidiu Horea Bedreag1,2, Marius Papurica1,2, Alexandru Florin Rogobete ${ }^{1,2}$, Dorel Sandesc ${ }^{1,2}$ \\ ${ }^{1}$ Clinic of Anaesthesia and Intensive Care, County Emergency Hospital, Timișoara, Romania \\ 2 Faculty of Medicine, "Victor Babeș" University of Medicine and Pharmacy, Timișoara, Romania
}

\section{CORRESPONDENCE}

Alexandru Florin Rogobete

Bd. Iosif Bulbuca nr. 10

300736 Timișoara, Romania

Tel: +40356433111

E-mail: alexandru.rogobete@umft.ro

\section{ARTICLE HISTORY}

Received: 10 July, 2016

Accepted: 20 July, 2016
Ovidiu Horea Bedreag • Bd. losif Bulbuca nr. 10 300736 Timișoara, Romania, Tel: +40 35643311 Marius Papurica • Bd. losif Bulbuca nr. 10, 300736 Timișoara, Romania, Tel: +40 356433111

Dorel Sandesc • Bd. losif Bulbuca nr. 10, 300736 Timișoara, Romania, Tel: +40 35643311

\begin{abstract}
Drowning in freshwater kills many people around the world. Complications are multiple and sometimes impossible to treat. Fluid and electrolyte resuscitation is difficult because of all the physiological, biophysical and biochemical changes that decrease the rate of survival. Extreme lung injury and cardiovascular disorders are responsible for tissue hypoxia, increased production of inflammation markers, biosynthesis of reactive oxygen species and finally, multiple organ damage. Hypothermia, frequently associated with drowning, provides multiple benefits to this type of patients. Various studies have developed the idea that hypothermia protects the brain from biochemical mediators, thereby preventing neuronal cell destruction. In this case report we present the biological parameters and evolution of a patient drowned in freshwater, and also the benefits of hypothermia to the clinical picture.
\end{abstract}

Keywords: drowning, inflammation, hypothermia, hypoxia, critical care unit

\section{INTRODUCTION}

Worldwide, annually, approximately 500,000 people die due to drowning in water. ${ }^{1}$ Drowning in fresh water leads to a number of complications that can dramatically decrease survival rates. Fresh water may pass from the alveoli into the blood to produce more complications - hemodilution due to the passage of water molecules through the alveolar-capillary interface and entering into circulation, increasing the circulating volume, leading to pulmonary and cerebral oedema. ${ }^{2,3}$

\section{CASE REPORT}

We present the case of P.R., female, weight $50 \mathrm{~kg}$, aged 33 years, blood group 0 I positive, received in the emergency room of the "Pius Brinzeu" County 

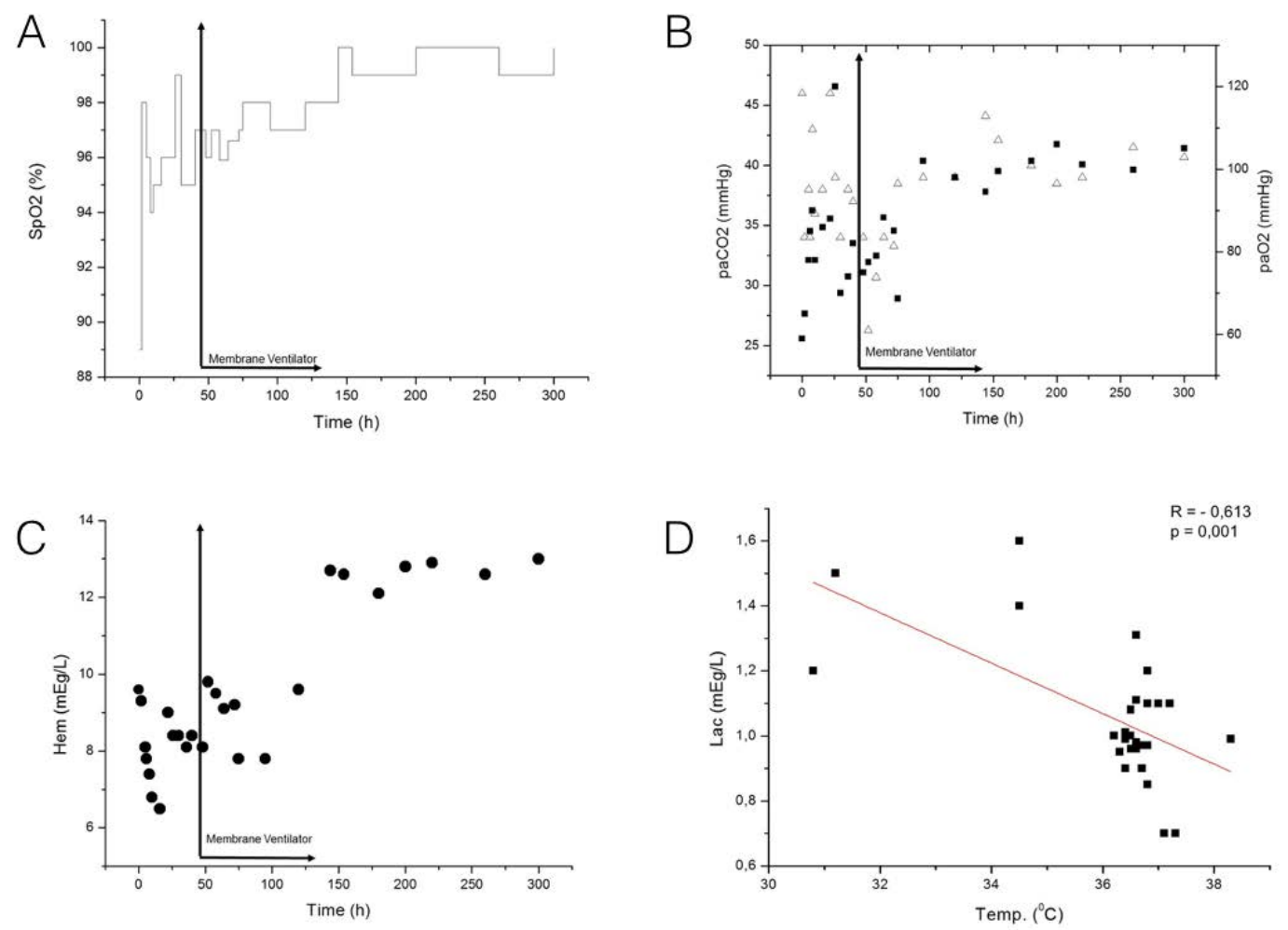

FIGURE 1. A $-\mathrm{SpO}_{2}$ dynamics. $\mathbf{B}-$ The evolution of partial pressure of $\mathrm{CO}_{2}$ and $\mathrm{O}_{2}$ during ICU admission $(\square)-\mathrm{mmHg} \mathrm{paO}_{2},(\Delta)-\mathrm{mmHg} \mathrm{paCO}_{2}$. C - The evolution of hemoglobin level during ICU admission.

D - Statistical correlation between lactic acid and temperature $(r=-0.613, p=0.001)$.

Emergency Hospital Timișoara, following a road traffic accident. According to the Timişoara Emergency Medical Service, the patient was immobilized in water for about 30 minutes (water temperature around $2^{\circ} \mathrm{C}$, ambient temperature around $-5^{\circ} \mathrm{C}$ ). When presenting to the accident, the extrication team found the victim immobilized between the front seats of the vehicle, without a seat belt and without oxygen supply. After extrication, the patient presented: heart rate (HR) $0 \mathrm{bpm}$, arterial pressure (AP) 0 mmHg, blood oxygen level $\left(\mathrm{SpO}_{2}\right) 0 \%$, mydriasis, unreactive pupils. Cardio-pulmonary resuscitation (CPR) lasted 25 minutes, after which the patient presented: HR $70 \mathrm{bpm}$, AP 100/70 mmHg, $\mathrm{SpO}_{2} 90 \%$ (endotracheal intubation), body temperature $30.6^{\circ} \mathrm{C}$.

At admission in the Intensive Care Unit (ICU) the patient was sedated, intubated endotracheally, mechanically ventilated - synchronized intermittent mandatory ventilation (SIMV) mode with conventional parameters, $\mathrm{SpO}_{2}$ 89\%, AP 96/62 mmHg, HR $130 \mathrm{bpm}$, severe hypothermia $\left(30.8^{\circ} \mathrm{C}\right)$ and macroscopic haematuria. Abdomen, head and pelvis CT were performed and electric heating blanket was established. After 24 hours of admission, weaning from the mechanical ventilator was started, but shortly after the patient needed reintubation. Chest $\mathrm{X}$-ray detected confluent opacities in both lung fields. Extracorporeal carbon dioxide removal and membrane oxygenation (ECMO) were started within $48 \mathrm{~h}$ of admission, after right femoral vein catheterization with continuous monitoring of acidbase balance. At the same time, mechanical ventilation was mantained to prevent lung tissue collapse.

The patient had a favorable evolution, after 120 hours she was extubated, breathing spontaneously and efficiently $\mathrm{O}_{2}$ on facial mask, $\mathrm{SpO}_{2} 99 \%$, with ECMO in place. After 130 hours, due to improved physiological and biological status, treatment with ECMO was stopped (Figure 1).

During the admission into the ICU, the pacient needed blood transfusion as a consequence of intensive macrohematuria and vaginal bleeding. Clinical evolution was favorable. In the ICU patient received clinical and paraclinical monitoring, imaging investigation (RX, MRI, CT), neurological, urological, gynecological, surgical and pharmacological review. After 300 hours, the patient was transfered to the Clinic of Neurology, showing improved clinical status.

\section{DISCUSSION}

A patient drowning in freshwater develops a series of complications that dramatically decrease the chance of survival 
due to biochemical and biophysical changes. Pulmonary disorders, acid-base and hydroelectrolyte imbalances ${ }^{2,4}$ may lead to systematic decompensation of the cardiovascular, respiratory, and gastrointestinal systems. ${ }^{4}$ All these combined lead to multiple organ dysfunction and death, due to the massive synthesis of reactive oxygen species, inflammatory mediators, and physiological and biochemical imbalance. ${ }^{4}$

Hypothermia plays an important role in the clinical evolution of the patient. The degree of hypothermia depends on several factors, such as: water temperature, wounds, metabolism, toxins etc. ${ }^{3,4}$ It is known that therapeutic hypothermia protects the brain from a series of injuries: stroke and brain injury, cardiac arrest, encephalopathy and spinal cord trauma, being more and more used in clinical practice. The protective effects of mild hypothermia are due to a decrease in basal metabolism, which leads to decreased levels of calcium ions and inflammation markers, decreased blood-brain barrier permeability, and inhibits the biosynthesis of reactive oxygen species. ${ }^{5}$ Also, it limits the consumption of glucose and oxygen, decreasing the risk of energy failure, and the biosynthesis of lactic acid in the blood and cerebrospinal fluid.

The inability of the respiratory system to provide gas exchange between the alveolar air and pulmonary capillaries is responsible for blood-gas imbalances. To avoid mechanical ventilation side effects by overdistension, cyclic collapse, alveolar damage, improper pressure or systemic inflammatory response, treatment with extracorporeal membrane oxygenation was started. Therefore, the associated lung damage was lower than with mechanical ventilation, and gas exchange increased $(r=-0.703$, $\mathrm{p}<0.001$ ), balancing the partial pressure of gases in the circulating blood (Figure 1). Regarding the electrolytes, the patient had mild hyponatremia in the first $24 \mathrm{~h}$ of admission and hypopotasemia after $96 \mathrm{~h}$ - both reaching normal values after intensive therapy (Figure 1). Lactate concentration was closely monitored. During hypothermia, lactate concentration was low, but after warming the patient and thus increasing the basal metabolism, the concentration reached a peak, which afterwards decreased and was maintained at a constant concentration. One of the side effects of ECMO is hemolytic anemia, therefore hemoglobin monitoring is necessary ( $\mathrm{r}=$ $0.864, \mathrm{p}<0.001)$. The patient presented severe hemolytic anemia, which was corrected by administration of blood products (Figure 1).

Pearson correlation coefficient ( $r$ ) and statistical significance using t-test were calculated. A p value $<0.05$ was considered statistically significant. To obtain graphics, the OriginPro 8 program was used (SR4 v.8.0951(B591), OriginLab Corporation). As previously presented, temperature has a direct influence on the concentration of lactic acid. This phenomenon is confirmed in our case report, the correlation being indirect and strongly significant $(\mathrm{r}=-0.613, \mathrm{p}<0.001)$. Once the $\mathrm{CO}_{2}$ removing extracorporeal membrane was started, there was an immediate improvement of arterial blood gases, especially $\mathrm{pH}$, which is strongly and indirectly correlated with the partial pressures of the gases $(r=-0.703, p<0.001)$ (Figure 1). The biological and biochemical test results emphasize the degree of hemolysis produced by the extracorporeal pump. Between the level of hemoglobin and the period of time the extracorporeal membrane was used, there is a significant and direct statistical correlation $(\mathrm{r}=0.864, \mathrm{p}<0.001)$. There were no significant statistical correlations between the other biological parameters.

\section{CONCLUSION}

Low water temperature $\left(2^{\circ} \mathrm{C}\right)$, immersion time $(30 \mathrm{~min})$, but also the ambient temperature during $\mathrm{CPR}\left(-5^{\circ} \mathrm{C}\right)$, all lead to severe hypothermia. Slowing the biochemical mechanisms, especially in the neurons, protected them from the lack of oxygen, lactate, energy failure, increased calcium concentration and ketone bodies. Treatment with extracorporeal membrane for oxygenation $/ \mathrm{CO}_{2}$ removal allowed rapid regeneration of pulmonary tissue and acid-base balance. Fluid and electrolyte resuscitation, intensive care, nursing and modern monitoring in the ICU have lead to improvement of the clinical status and the patient's survival.

\section{CONFLICT OF INTEREST}

Nothing to declare.

\section{REFERENCES}

1. Yajima D, Saito H, Sato K, Hayakawa M, Iwase $H$. Diagnosis of drowning by summation of sodium, potassium and chloride ion levels in pleural effusion: differentiating between freshwater and seawater drowning and application to bathtub deaths. Forensic Sci Int. 2013;233:167-173.

2. Usumoto $Y$, Sameshima N, Hikiji W, et al. Electrolyte analysis of pleural effusion as an indicator of drowning in seawater and freshwater. $J$ Forensic Leg Med. 2009;16:321-324.

3. Lucci A, Campobasso CP, Cirnelli A, Lorenzini G. A promising microbiological test for the diagnosis of drowning. Forensic Sci Int. 2008;182:20-26.

4. Zhang M, Zhou GJ, Zhao S, Yang JX, Lu X, Gan JX, et al. Delayed diagnosis of tooth aspiration in three multiple trauma patients with mechanical ventilation. Crit Care. 2011:15:424

5. Pérez-Cárceles MD, del Pozo S, Sibón A, et al. Serum biochemical markers in drowning: diagnostic efficacy of Strontium and other trace elements. Forensic Sci Int. 2012;214:159-166. 